\title{
Acute seizure activity promotes lipid peroxidation, increased nitrite levels and adaptive pathways against oxidative stress in the frontal cortex and striatum
}

\author{
Hélio Vitoriano Nobre Júnior, ${ }^{1}$ Marta Maria de França Fonteles ${ }^{2}$ and Rivelilson Mendes de Freitas ${ }^{3, *}$
}

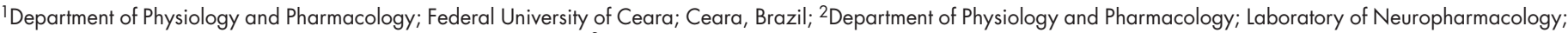
School of Medicine; Federal University of Ceara; Ceara, Brazil; ${ }^{3}$ Department of Physiology and Pharmacology; Federal University of Piaui; Picos, Piauí Brazil

Abbreviations: SE, status epilepticus; GSH, glutathione reduced; 24 h, 24 hours; TLE, temporal lobe epilepsy; OS, oxidative stress; $\mathrm{O}_{2}^{-}$, superoxide; $\mathrm{OH}$, hydroxyl radical; $\mathrm{NO}$, nitric oxide; $\mathrm{H}_{2} \mathrm{O}_{2}$, hydrogen peroxide; $\mathrm{O}_{2}$, oxygen; $\mathrm{H}_{2} \mathrm{O}$, water; $\mathrm{SOD}$, superoxide dismutase; ROS, reactive oxygen species; TBARS, thiobarbituric-acid-reacting substances; MDA, malondialdehyde; $\mathrm{H}_{3} \mathrm{PO}_{4}$, phosphoric acid; $\mathrm{NaNO}_{2}$, sodium nitrate; EDTA, ethylenediamine tetraacetic acid; KCN, potassium cyanide; CAT, catalase; SEM, standard error of the mean

Key words: lipid peroxidation, nitrite content, glutathione reduced, catalase, superoxide dismutase, striatum, frontal cortex

Previous experiments have shown that the generation of free radicals in rat brain homogenates is increased following pilocarpine-induced seizures and status epilepticus (SE). This study was aimed at investigating the changes in neurochemical mechanisms such as lipid peroxidation levels, nitrite content, glutathione reduced (GSH) concentration, superoxide dismutase and catalase activities in the frontal cortex and the striatum of Wistar adult rats after seizures and SE induced by pilocarpine. The control group was treated with $0.9 \%$ saline and another group of rats received pilocarpine $(400 \mathrm{mg} / \mathrm{kg}$, i.p.). Both groups were sacrificed $24 \mathrm{~h}$ after the treatments. Lipid peroxidation level, nitrite content, GSH concentration and enzymatic activities were measured by using spectrophotometric methods. Our findings showed that pilocarpine administration and its resulting seizures and SE produced a significant increase of lipid peroxidation level in the striatum (47\%) and frontal cortex (59\%). Nitrite contents increased $49 \%$ and $73 \%$ in striatum and frontal cortex in pilocarpine group, respectively. In GSH concentrations were decreases of $54 \%$ and $58 \%$ in the striatum and frontal cortex in pilocarpine group, respectively. The catalase activity increased $39 \%$ and $49 \%$ in the striatum and frontal cortex, respectively. The superoxide dismutase activity was not altered in the striatum, but it was present at a $24 \%$ increase in frontal cortex. These results suggest that there is a direct relationship between

*Correspondence to: Rivelilson Mendes de Freitas; Department of Physiology and Pharmacology; Federal University of Piaui; Rua Cícero Eduardo, s/n, Junco; Picos, Piauí 63900-00 Brazil; Tel./Fax: 55.89.3422.4389; Email: rivelilson@ufpi.br

Submitted: 03/01/09; Revised: 03/12/09; Accepted: 03/19/09

Previously published online as an Oxidative Medicine and Cellular Longevity E-publication:

http://www.landesbioscience.com/journals/oximed/article/8488 the lipid peroxidation and nitrite contents during epileptic activity that can be responsible for the superoxide dismutase and catalase enzymatic activity changes observed during the establishment of seizures and SE induced by pilocarpine.

\section{Introduction}

Numerous neurochemical studies using animals have revealed that oxidative stress-related seizures produce changes in antioxidant enzymatic activity and receptor binding. ${ }^{1-3}$ Oxidative stress-related brain disorders, including epilepsy, showed a substantial decrease in cholinergic markers, such as acetylcholinesterase ${ }^{4}$ and a number of muscarinic receptors in the hippocampus, striatum and frontal cortex of adult rats. ${ }^{5,6}$ Epilepsy is a complex neurobehavioral disorder that can be the result of increased excitability of neurons in several brain regions. ${ }^{7,8}$ Pilocarpine administration makes it progresse to a long-lasting SE within $1-2 \mathrm{~h}$ and induces behavioral and electroencephalographic alterations which are similar the TLE in humans. ${ }^{9}$ Epilepsy model induced by pilocarpine in rodents can provide information regarding oxidative stress-related epileptic activity. ${ }^{10-12}$ TLE can be characterized by a permanent change in OS that is more facilitated in the brain in relation to others tissues because it contains large quantities of oxidizable lipids and metals and a lower number of antioxidant defenses. ${ }^{13,14}$

Oxidative stress-related seizures can be defined by excessive production of free radicals, such as $\mathrm{O}_{2}^{-}, \mathrm{OH}, \mathrm{NO}$ and their metabolites (nitrate and nitrite) and other ones, which can dramatically alter the neuronal function. In addition, an increase in production of these compounds has been related to neuronal death induced by seizures. ${ }^{13,15,16}$ Several compounds can produce free radicals such as $\mathrm{H}_{2} \mathrm{O}_{2}$, which in high concentration can react with $\mathrm{O}_{2}{ }^{-}$(HaberWeiss reaction) or iron (Fenton reaction) producing the highly 
reactive hydroxyl radical. The conversion of $\mathrm{H}_{2} \mathrm{O}_{2}$ to $\mathrm{H}_{2} \mathrm{O}$ and $\mathrm{O}_{2}$ is made by catalase and glutathione peroxidase. ${ }^{27,18}$ The formed hydroxyl radical is likely to react with non-radical molecules, transforming them into secondary free radicals. This reaction takes place during the lipid peroxidation producing hydroperoxides. ${ }^{14,17}$ The NO also produced during seizures can be estimated by their metabolites (nitrite and nitrate). ${ }^{19,20}$ Despite the fact that numerous studies clearly indicate the importance of antioxidant enzymatic activities during epileptic phenomenon the mechanisms by which these enzymes play role in installation and/or propagation of seizures and SE are not completely understood. ${ }^{17,18}$

In the brain, the phenomena of excitotoxicity has been related to an over production of free radicals by the neurons during seizures and SE induced by pilocarpine ${ }^{18}$ as well as in brain of epileptic patients. ${ }^{19}$ The increase of reactive oxygen species levels can be responsible by neurochemical alterations observed during seizures. ${ }^{16}$ Studies have suggested an increase in antioxidant enzymatic activity, such as SOD and catalase, during pilocarpineinduced seizures, indicating a neuronal response against the oxidative stress-related seizures. ${ }^{16}$ An elevation in content of ROS during seizures can induce an increase in contents of oxidized proteins, ${ }^{21}$ nitrite ${ }^{7}$ and lipid peroxidation. ${ }^{22}$ This work was performed to investigate lipid peroxidation level, nitrite content, GSH concentration, superoxide dismutase and catalase activities in the striatum and frontal cortex of adult rats during acute phase of seizures induced by pilocarpine.

\section{Results}

Behavioral alterations after treatment with pilocarpine. According to our previous studies, few minutes after pilocarpine administration, all animals presented stereotyped oral and masticatory movements, hypokinesia, salivation, tremor and partial or generalized limbic seizures. ${ }^{9,23,24}$ Approximately 35 min following pilocarpine injection, the animals presented seizures that develop to $\mathrm{SE}$ for over $30 \mathrm{~min}$. During the $24 \mathrm{~h}$ observation period, $60 \%$ of animals died.

Lipid peroxidation level in the striatum and frontal cortex of adult rats after pilocarpine-induced seizures. A literature review revealed that there are articles that address the role of oxidative stress in neurological disorders, including seizure models induced by pilocarpine in which the modulation of the pro-oxidant/ antioxidant balance by seizures per se and by antioxidant agents is discussed. ${ }^{1,2,25}$ However, the critical role of oxidative stress in this seizure models is not uniform. Therefore, there is a need for a study that will address these issues. The lipid peroxidation (TBARS formed) in the brain homogenates was markedly increased in this model as compared to corresponding values for the control group. After pilocarpine-induced SE, significant increases of $47 \%$ and $59 \%$ in the striatum $[t(10)=11.820$; $\mathrm{p}<0.0001]$ and in frontal cortex $[t(11)=23.744 ; \mathrm{p}<0.0001]$ in TBARS levels were presented, respectively, as compared to control group (Figs. 1 and 2).

Nitrite content in the striatum and frontal cortex of adult rats after pilocarpine-induced seizures. SE produced marked increases in nitrite content -49 and $73 \%$ in the striatum $[t(18)=10.871$;

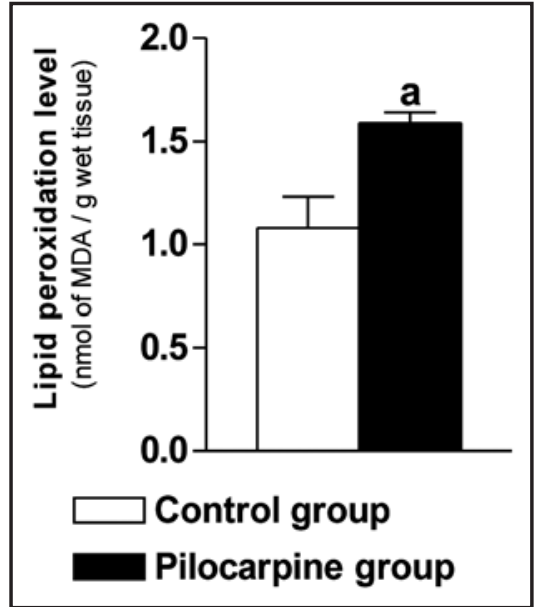

Figure 1. Lipid peroxidation level is increased in the striatum during seizure activity. Male rats (250-280 g, 2-months-old) were treated with a single dose of pilocarpine (400 $\mathrm{mg} / \mathrm{kg}$, i.p., $n=7$, pilocarpine group) and the control group with $0.9 \%$ saline $(n=9)$. The pilocarpine group was constituted by those rats that presented seizures, SE for over $30 \mathrm{~min}$ and that did not died within $24 \mathrm{~h}$. For neurochemical determinations of lipid peroxidation level, the rats (pilocarpine and control groups) were killed by decapitation $24 \mathrm{~h}$ after the treatment and their brains were dissected on ice to remove the striatum. The cerebral area studied was ultrasonically homogenized in $1 \mathrm{ml}$ of $0.05 \mathrm{M}$ phosphate buffer, $\mathrm{pH} 7.0$, and the homogenates $10 \%$ were centrifuged $(800 \mathrm{~g} / 20 \mathrm{~min})$ and the supernatant of the homogenates was used for lipid peroxidation level investigation. The lipid peroxidation level in the striatum during seizure activity was analyzed by measuring the thiobarbituric-acid-reacting substances (TBARS) in homogenates, as previously described by Draper and Hadley, 1990. Results are expressed as means + S.E.M. for the number of animals shown inside in parenthesis. Results were expressed as nmol of malondialdehyde $(M D A) / g$ wet tissue. The Student-Newman-Keuls test was used for multiple comparisons of means of two groups of data. Differences in experimental groups were determined by two-tailed analysis of variance. Differences were considered significant at $p<0.05$. ${ }^{a} p<0.05$ as compared with control group (analysis of variance and Student-Newman-Keuls test).

$\mathrm{p}<0.0001]$ and in frontal cortex $[t(18)=30.284 ; \mathrm{p}<0.0001]$, respectively, as compared to control group (Figs. 3 and 4).

GSH concentration in the striatum and frontal cortex of adult rats after pilocarpine-induced seizures. GSH concentrations had important decreases of 54 and $58 \%$ in the striatum $[t(10)=60.660 ; \mathrm{p}<0.0001]$ and in frontal cortex $[t(10)=54.580 ; \mathrm{p}<0.0001]$ of adult rats after pilocarpineinduced seizures, respectively, as compared to the control group (Figs. 5 and 6).

Superoxide dismutase and catalase activities in the striatum and frontal cortex of adult rats after pilocarpine-induced seizures. Superoxide dismutase activity in the striatum [ $t(16)$ $=0.1540 ; p=$ N.S.] after seizures did not presented any difference statistically significant, however, in frontal cortex it was verified an increase of $24 \%$ after seizures $[t(16)=2.921$; $\mathrm{p}<0.0100]$ as compared to the control group (Fig. 7). In catalase activity after SE, it was verified increases of 39 and $49 \%$ in the striatum $[t(16)=6.979 ; \mathrm{p}<0.0001]$ and in frontal cortex $[t(16)=6.609 ; \mathrm{p}<0.0001]$, respectively, as compared to the control group (Fig. 8). 


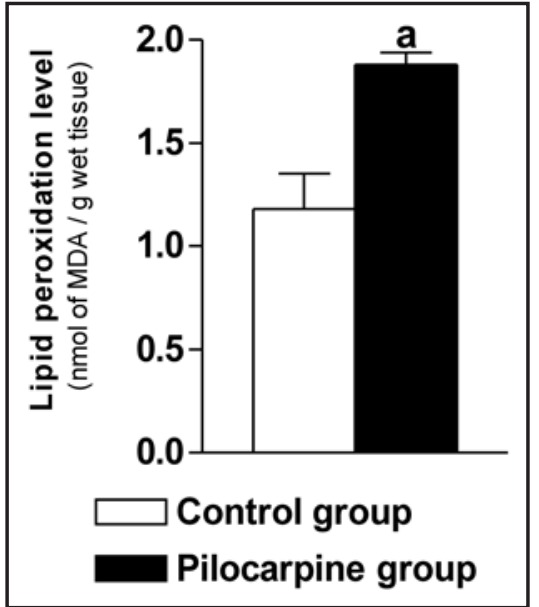

Figure 2. Lipid peroxidation level is increased in the frontal cortex during seizure activity. Male rats (250-280 g, 2-months-old) were treated with a single dose of pilocarpine (400 mg/ $\mathrm{kg}$, i.p., $n=7$, pilocarpine group) and the control group with $0.9 \%$ saline $(n=9)$. The pilocarpine group was constituted by those rats that presented seizures, SE for over 30 min and that did not died within $24 \mathrm{~h}$. For neurochemical determinations of lipid peroxidation level, the rats (pilocarpine and control groups) were killed by decapitation $24 \mathrm{~h}$ after the treatment and their brains were dissected on ice to remove the striatum. The cerebral area studied was ultrasoni-

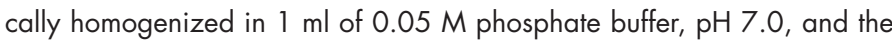
homogenates $10 \%$ were centrifuged $(800 \mathrm{~g} / 20 \mathrm{~min})$ and the supernatant of the homogenates was used for lipid peroxidation level investigation. The lipid peroxidation level in the striatum during seizure activity was analyzed by measuring the thiobarbituric-acid-reacting substances (TBARS) in homogenates, as previously described by Draper and Hadley, 1990. Results are expressed as means + S.E.M. for the number of animals shown inside in parenthesis. Results were expressed as nmol of malondialdehyde (MDA)/g wet tissue. The Student-Newman-Keuls test was used for multiple comparisons of means of two groups of data. Differences in experimental groups were determined by two-tailed analysis of variance. Differences were considered significant at $p<0.05$. ${ }^{a} p<0.05$ as compared with control group (analysis of variance and Student-Newman-Keuls test).

\section{Discussion}

Neuronal hyperexcitability and excessive production of free radicals have been implicated in the pathogenesis of a considerable range of neurological disorders, including epilepsy. Our results for the behavioral alterations observed after pilocarpine administration match with previous data described. ${ }^{23}$ Lipid peroxidation in a tissue is an index of irreversible biological damage of the cell membrane phospholipid, which in turn leads to inhibition of most of the sulphydryl and some nonsulphydryl enzymes. ${ }^{26,27}$ Lipid peroxidation can be induced by many chemicals (e.g., kainic acid and pilocarpine) and by many tissue injuries, and it has been suggested as a possible mechanism for the neurotoxic effects observed during epileptic activity. ${ }^{7,26}$ Our findings demonstrated that lipid peroxidation levels in the striatum and frontal cortex of adult rats were increased after 24 of the acute phase of seizures induced by pilocarpine. Literature suggests that after febrile seizures in children the erythrocyte malondialdehyde levels were significantly elevated, reaffirming the hypothesis of lipid peroxidation produced during seizure activity. ${ }^{28}$

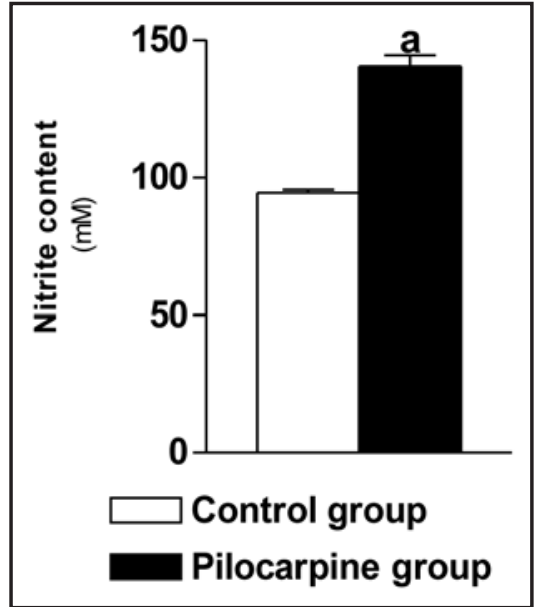

Figure 3. Nitrite content is increased in the striatum during seizure activity. Male rats (250-280 g, 2-months-old) were treated with a single dose of pilocarpine (400 mg/kg, i.p., $n=7$, pilocarpine group) and the control group with $0.9 \%$ saline $(n=9)$. The pilocarpine group was constituted by those rats that presented seizures, SE for over $30 \mathrm{~min}$ and that did not died within $24 \mathrm{~h}$. For neurochemical determinations of lipid peroxidation level, the rats (pilocarpine and control groups) were killed by decapitation $24 \mathrm{~h}$ after the treatment and their brains were dissected on ice to remove the striatum. The cerebral area studied was ultrasonically homogenized in

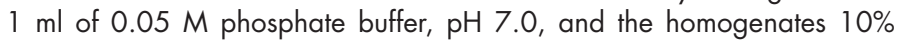
were centrifuged $(800 \mathrm{~g} / 20 \mathrm{~min})$ and the supernatant of the homogenates was used for lipid peroxidation level investigation. The lipid peroxidation level in the striatum during seizure activity was analyzed by measuring the thiobarbituric-acid-reacting substances (TBARS) in homogenates, as previously described by Draper and Hadley, 1990. Results are expressed as means + S.E.M. for the number of animals shown inside in parenthesis. Results were expressed as nmol of malondialdehyde (MDA)/g wet tissue. The Student-Newman-Keuls test was used for multiple comparisons of means of two groups of data. Differences in experimental groups were determined by two-tailed analysis of variance. Differences were considered significant at $p<0.05$. ${ }^{a} p<0.05$ as compared with control group (analysis of variance and Student-Newman-Keuls test).

In normal conditions, there is a steady state balance between the ROS production and their scavenging by the cellular antioxidant system. We demonstrated that the nitrite content in the striatum and frontal cortex of adult rats was augmented after seizures and SE induced by pilocarpine, suggesting a possible increase in the ROS level which can be involved in the neuronal damage induced by seizures. Other studies have shown that nitrite levels in the hippocampus were elevated during seizures. ${ }^{3}$ Thus, it is tempting to speculate that during epileptic activity there is an increase in ROS content. ROS levels may be associated with the pathogenesis of various pediatric central nervous system diseases, including epilepsy. ${ }^{29}$ We also demonstrated that the glutathione reduced concentration in the striatum and frontal cortex after seizure activity was decreased, suggesting that its compounds play a role neuroprotective function against neuronal damage produced by increase in the ROS level. The data indicate that the ROS accumulation in GSH deficiency decreases antioxidant capacity in the striatum and frontal cortex. Therefore it is presumed that this pathomecanism may contribute at least in part to the pathophysiology of the seizure activity. Other studies showed 


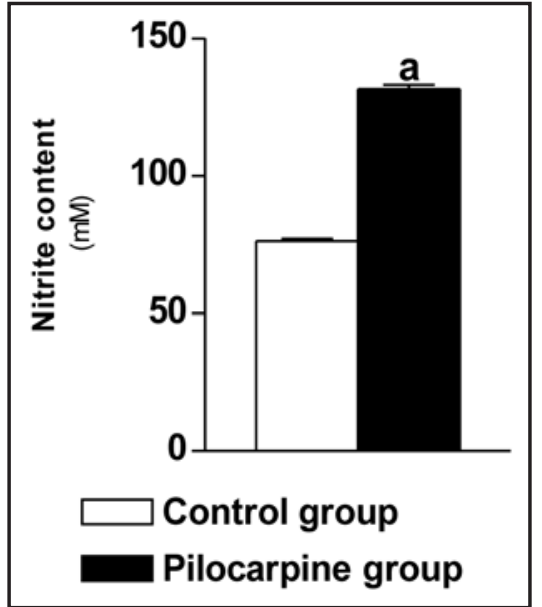

Figure 4. Nitrite content is increased in the frontal cortex during seizure activity. Male rats (250-280 g, 2-months-old) were treated with a single dose of pilocarpine (400 mg/kg, i.p., $n=7$, pilocarpine group) and the control group with $0.9 \%$ saline $(n=9)$. The pilocarpine group was constituted by those rats that presented seizures, SE for over 30 min and that did not died within $24 \mathrm{~h}$. For neurochemical determinations of lipid peroxidation level, the rats (pilocarpine and control groups) were killed by decapitation $24 \mathrm{~h}$ after the treatment and their brains were dissected on ice to remove the striatum. The cerebral area studied was ultrasonically homogenized in $1 \mathrm{ml}$ of $0.05 \mathrm{M}$ phosphate buffer, $\mathrm{pH} 7.0$, and the homogenates $10 \%$ were centrifuged $(800 \mathrm{~g} / 20 \mathrm{~min}$ ) and the supernatant of the homogenates was used for lipid peroxidation level investigation. The lipid peroxidation level in the striatum during seizure activity was analyzed by measuring the thiobarbituric-acid-reacting substances (TBARS) in homogenates, as previously described by Draper and Hadley, 1990. Results are expressed as means + S.E.M. for the number of animals shown inside in parenthesis. Results were expressed as nmol of malondialdehyde (MDA)/g wet tissue. The Student-Newman-Keuls test was used for multiple comparisons of means of two groups of data. Differences in experimental groups were determined by two-tailed analysis of variance. Differences were considered significant at $p<0.05$. ${ }^{a} p<0.05$ as compared with control group (analysis of variance and Student-Newman-Keuls test).

that neurometabolic disorders produces a decrease in antioxidant defenses in the striatum ${ }^{30}$ and hippocampus of rats after seizures. ${ }^{3}$ However, further studies using antioxidants drugs during seizures and SE induced by pilocarpine can indicate whether lipid peroxidation and nitrite concentrations play role in the pathophysiology of installation and/or propagation of the limbic seizures.

Oxidative stress can occur in all tissues, yet the animal brain is often said to be especially sensitive. ${ }^{31,32}$ Oxidative stress, resulting from excitotoxicity, is implicated in numerous neurodegenerative conditions. Damage due to superoxide, hydroxyl radical and peroxynitrite has been observed in epilepsy. Antioxidant therapies to remove these toxic compounds have been of great interest in the treatment of these disorders. ${ }^{33}$ The outcomes of our experiments demonstrate that pilocarpine administration and its resulting seizures and SE induces significant alterations in antioxidant defenses in the striatum and frontal cortex of adult rats. We recorded alterations in the superoxide dismutase activity in the frontal cortex during the seizures and SE, however, under the same conditions, the striatal superoxide dismutase activity remained constant. It is likely that the unaltered superoxide dismutase

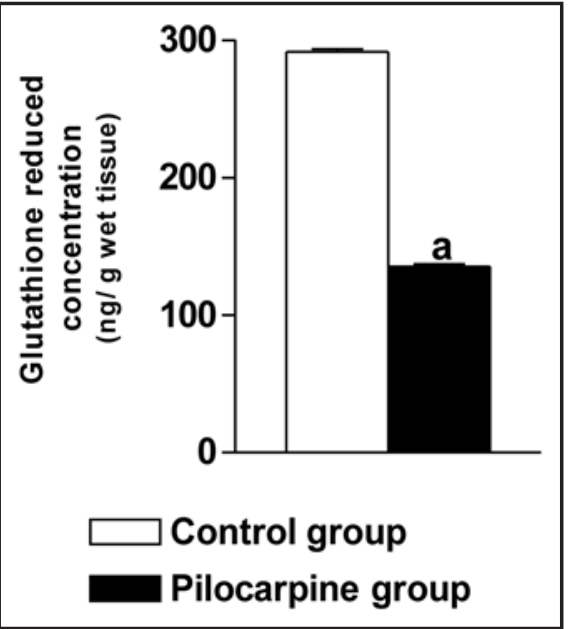

Figure 5. Glutathione reduced concentration is decreased in the striatum during seizure activity. Male rats (250-280 g, 2-months-old) were treated with a single dose of pilocarpine $(400 \mathrm{mg} / \mathrm{kg}$, i.p., $n=7$, pilocarpine group) and the control group with $0.9 \%$ saline $(n=9)$. The pilocarpine group was constituted by those rats that presented seizures, SE for over 30 min and that did not died within $24 \mathrm{~h}$. For neurochemical determinations of lipid peroxidation level, the rats (pilocarpine and control groups) were killed by decapitation $24 \mathrm{~h}$ after the treatment and their brains were dissected on ice to remove the striatum. The cerebral area studied was ultrasonically homogenized in $1 \mathrm{ml}$ of $0.05 \mathrm{M}$ phosphate buffer, $\mathrm{pH} 7.0$, and the homogenates $10 \%$ were centrifuged $(800 \mathrm{~g} / 20 \mathrm{~min})$ and the supernatant of the homogenates was used for lipid peroxidation level investigation. The lipid peroxidation level in the striatum during seizure activity was analyzed by measuring the thiobarbituric-acid-reacting substances (TBARS) in homogenates, as previously described by Draper and Hadley, 1990. Results are expressed as means + S.E.M. for the number of animals shown inside in parenthesis. Results were expressed as $\mathrm{nmol}$ of malondialdehyde (MDA)/g wet tissue. The Student-NewmanKeuls test was used for multiple comparisons of means of two groups of data. Differences in experimental groups were determined by two-tailed analysis of variance. Differences were considered significant at $p<0.05$. ${ }^{a} p<0.05$ as compared with control group (analysis of variance and Student-Newman-Keuls test).

activity in the striatum might not be modified during the oxidative stress-related mechanisms involved in the installation and propagation of seizures and SE induced by pilocarpine, which produces several changes in parameters related to generation and elimination of oxygen free radicals in adult rats. ${ }^{18}$ An increase in free radical formation can be accompanied by an immediate compensatory increase in free radical scavenging enzymatic activities and this action was observed during seizures and SE in the frontal cortex. Nevertheless, a similar compensatory mechanism of scavenging was observed only in striatal catalase activity after seizures and SE, suggesting that the enzymatic function of different systems can be modified either during the acute phase of seizures or according to cerebral area investigated. In addition, it has been demonstrated that studies performed with antioxidant compounds (vitamin $\mathrm{C}$, vitamin $\mathrm{E}$ and lipoic acid) produces an increase in catalase activity in the hippocampus of rats after seizures, which suggests a neuroprotective action of these compounds through brain modulation of antioxidant enzymatic activity. ${ }^{1,2,34}$ 


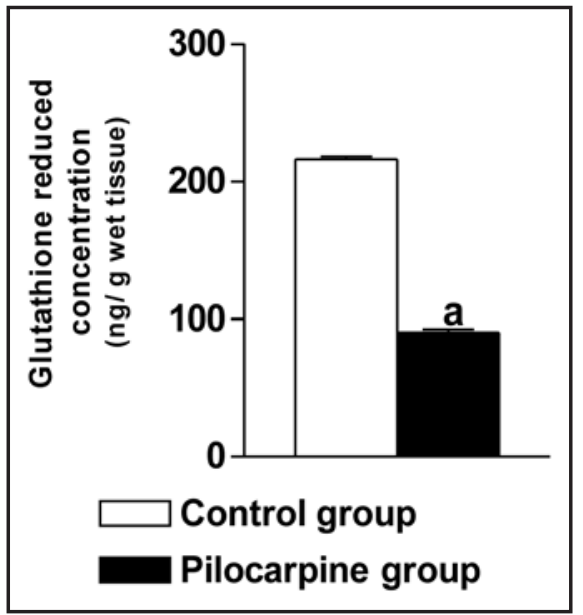

Figure 6. Glutathione reduced concentration is decreased in the frontal cortex during seizure activity. Male rats (250-280 g, 2-months-old) were treated with a single dose of pilocarpine $(400 \mathrm{mg} / \mathrm{kg}$, i.p., $n=7$, pilocarpine group) and the control group with $0.9 \%$ saline $(n=9)$. The pilocarpine group was constituted by those rats that presented seizures, SE for over $30 \mathrm{~min}$ and that did not died within $24 \mathrm{~h}$. For neurochemical determinations of lipid peroxidation level, the rats (pilocarpine and control groups) were killed by decapitation $24 \mathrm{~h}$ after the treatment and their brains were dissected on ice to remove the striatum. The cerebral area studied was ultrasonically homogenized in $1 \mathrm{ml}$ of $0.05 \mathrm{M}$ phosphate buffer, $\mathrm{pH} 7.0$, and the homogenates $10 \%$ were centrifuged $(800 \mathrm{~g} / 20 \mathrm{~min}$ ) and the supernatant of the homogenates was used for lipid peroxidation level investigation. The lipid peroxidation level in the striatum during seizure activity was analyzed by measuring the thiobarbituric-acid-reacting substances (TBARS) in homogenates, as previously described by Draper and Hadley, 1990. Results are expressed as means + S.E.M. for the number of animals shown inside in parenthesis. Results were expressed as $\mathrm{nmol}$ of malondialdehyde (MDA)/g wet tissue. The Student-Newman-Keuls test was used for multiple comparisons of means of two groups of data. Differences in experimental groups were determined by two-tailed analysis of variance. Differences were considered significant at $p<0.05$. ${ }^{a} p<$ 0.05 as compared with control group (analysis of variance and StudentNewman-Keuls test).

The present work reports the involvement of catalase activity in the striatum and frontal cortex after $24 \mathrm{~h}$ of the seizures and SE. An increase in the catalase activity in these brain areas can be related to a long-term compensatory mechanism including modulation in activity of enzymes responsible by ROS catabolism. Moreover, the catalase activity might be one of the mechanisms that avoid the development of neurotoxic effects mediated by seizures and SE, indicating that basal-oxygen radical production can damage the cell and that its control is necessary. ${ }^{14,17}$

Evidences for the role of free radicals in seizures have been found by using antioxidants drugs for protection against seizures and SE-induced neuronal damage. ${ }^{1,2,35}$ A steady level of $\mathrm{O}_{2}{ }^{-}$and $\mathrm{H}_{2} \mathrm{O}_{2}$ is always present within neurons as a result of a normal metabolism. SOD and catalase are responsible for degradation of $\mathrm{O}_{2}{ }^{-}$and $\mathrm{H}_{2} \mathrm{O}_{2}$, respectively. The balance between antioxidants enzymes, superoxide dismutase and catalase can be important during seizures and SE induced by pilocarpine. The present findings indicate that pilocarpine administration and its resulting seizures and SE induces neurochemical changes such as an increase

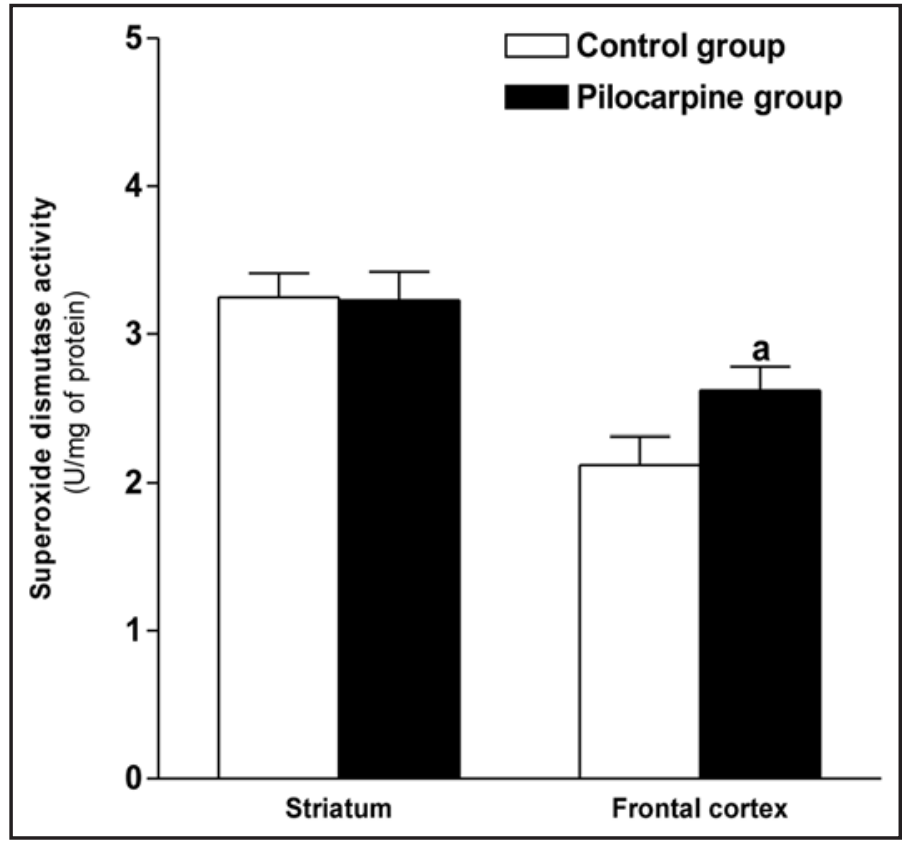

Figure 7. Superoxide dismutase activity is increased in the frontal cortex during seizure activity. Male rats (250-280 g, 2-months-old) were treated with a single dose of pilocarpine $(400 \mathrm{mg} / \mathrm{kg}$, i.p., $n=7$, pilocarpine group) and the control group with $0.9 \%$ saline $(n=9)$. The pilocarpine group was constituted by those rats that presented seizures, SE for over 30 min and that did not died within $24 \mathrm{~h}$. For neurochemical determinations of lipid peroxidation level, the rats (pilocarpine and control groups) were killed by decapitation $24 \mathrm{~h}$ after the treatment and their brains were dissected on ice to remove the striatum. The cerebral area studied was ultrasonically homogenized in $1 \mathrm{ml}$ of $0.05 \mathrm{M}$ phosphate buffer, $\mathrm{pH}$ 7.0 , and the homogenates $10 \%$ were centrifuged $(800 \mathrm{~g} / 20 \mathrm{~min})$ and the supernatant of the homogenates was used for lipid peroxidation level investigation. The lipid peroxidation level in the striatum during seizure activity was analyzed by measuring the thiobarbituric-acid-reacting substances (TBARS) in homogenates, as previously described by Draper and Hadley, 1990. Results are expressed as means + S.E.M. for the number of animals shown inside in parenthesis. Results were expressed as nmol of malondialdehyde (MDA)/g wet tissue. The Student-Newman-Keuls test was used for multiple comparisons of means of two groups of data. Differences in experimental groups were determined by two-tailed analysis of variance. Differences were considered significant at $p<0.05$. ${ }^{a} p<$ 0.05 as compared with control group (analysis of variance and StudentNewman-Keuls test).

in nitrite content and in lipid peroxidation level as well as an activation of cerebral antioxidant mechanisms. The anatomic distribution of alterations observed in the enzymatic activities (superoxide dismutase and catalase) can suggest that the frontal cortex can be extensively involved in the propagation of epileptic activity and further studies should be carried out to ascertain that catabolism of free radicals can be involved in the pathogenesis of seizures.

The pilocarpine model is essential to investigate the mechanisms for initiation and propagation of seizures and SE. Additionally, it may be assumed that the increased generation of nitrite and lipid peroxidation levels after seizures and SE is not primarily caused by an exhaustion of both the defense systems measured. 
Adaptative mechanisms, as the induction of catalase activity, may be taken into consideration to counteract oxidative stress mediated by seizures and SE. However, the relation among brain structures, antioxidant systems, lipid peroxidation level, nitrite concentration and seizures cannot be perfectly established and deserve further investigation.

\section{Materials and Methods}

Animals and experimental procedures. Male Wistar rats (250-280 g; 2-months-old) were used. Animals were housed in cages with free access to food and water. All animals were kept under standard light-dark cycle (lights on at 07:00 h a.m.). The experiments were performed according to the Guide for the care and use of laboratory of the US Department of Health and Human Services, Washington, DC (1985). Control animals received $0.9 \%$ saline intraperitonealy (i.p.) (Control group; $n=$ 47) and in the experimental group, the animals were treated with a single dose of pilocarpine hydrochloride $(400 \mathrm{mg} / \mathrm{kg}$, i.p.; $n=$ 43). Behavioral changes were observed for $24 \mathrm{~h}$. The parameters assessed were: number of peripheral cholinergic signs, tremors, stereotyped movements, seizures, SE and mortality. The SE was defined as continuous seizures for a period longer than $30 \mathrm{~min}$. SE was induced by method described by Turski et al. ${ }^{9}$ Mortality was recorded for $24 \mathrm{~h}$ after pilocarpine-induced seizures and corresponded to $60 \%$. The pilocarpine group studied was constituted by those rats that presented seizures, SE for a period longer than 30 min and that did not died within $24 \mathrm{~h}$ of observation.

For neurochemical determinations (lipid peroxidation level, nitrite content, GSH concentration, superoxide dismutase and catalase activities), the rats (pilocarpine and control groups) were killed by decapitation after $24 \mathrm{~h}$ from the treatment and their brains were dissected on ice to remove the frontal cortex and striatum. The pilocarpine group $(n=43)$ was constituted by those rats that presented seizures, SE for a period longer than $30 \mathrm{~min}$ and that did not died during $24 \mathrm{~h}$. Detailed criteria to determine these periods after pilocarpine administration were reported previously by Cavalheiro et al. ${ }^{36}$

Lipid peroxidation level determination in the striatum and frontal cortex during seizure activity. A study carried out by Walz et al., ${ }^{37}$ showed that after status epilepticus induced by pilocarpine can be observed an increase in lipid peroxidation level in hippocampus of Wistar rats. Based in this fact, we decided investigate the lipid peroxidation level in other areas involved in seizure activity. For all of the experimental procedures studied, homogenates $10 \%(\mathrm{w} / \mathrm{v})$ of each area investigated were prepared for both groups. The homogenates $10 \%$ were centrifuged $(800 \mathrm{~g} / 20 \mathrm{~min})$ and the supernatant of the homogenates was used for lipid peroxidation level investigation. The lipid peroxidation process in the pilocarpine group $(n=7)$ and control animals $(n=9)$ was analyzed by measuring the thiobarbituric-acid-reacting substances (TBARS) in homogenates, as previously described by Draper and Hadley. ${ }^{38}$ Briefly, the samples were mixed with $1 \mathrm{ml}$ of trichloroacetic acid $10 \%$ and $1 \mathrm{ml}$ of thiobarbituric acid $0.67 \%$ then heated in a boiling water bath for $15 \mathrm{~min}$, and butanol $(2: 1 \mathrm{v} / \mathrm{v})$ was added to the solution. After a centrifugation

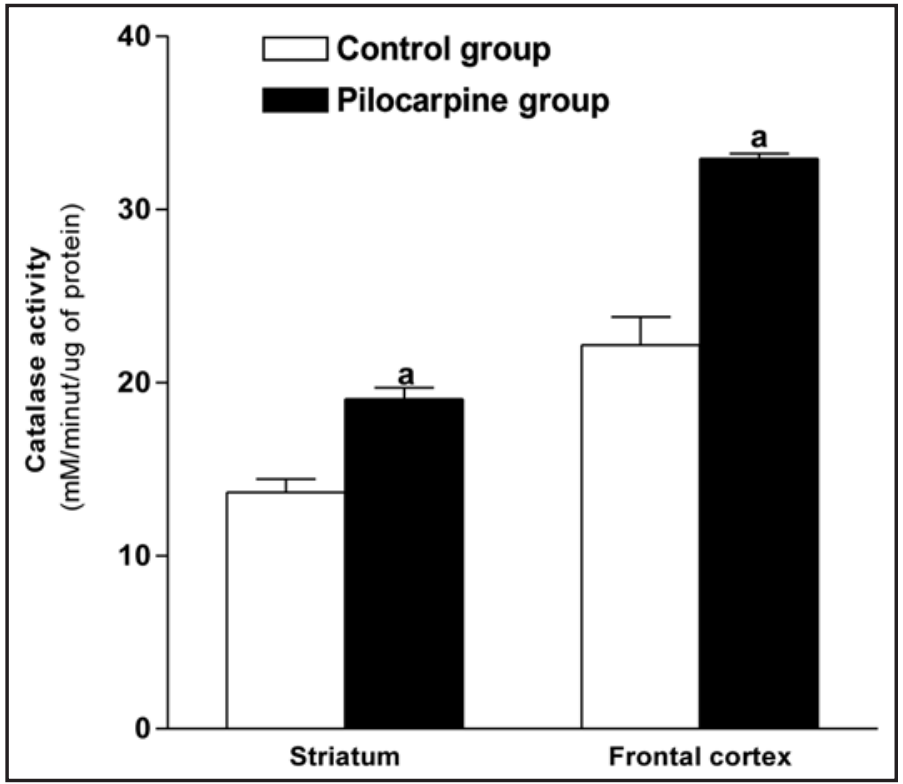

Figure 8. Catalase activity is increased in the striatum and frontal cortex during seizure activity. Male rats (250-280 g, 2-month-old) were treated with a single dose of pilocarpine $(400 \mathrm{mg} / \mathrm{kg}$, i.p., $n=7$, pilocarpine group) and the control group with $0.9 \%$ saline $(n=9)$. The pilocarpine group was constituted by those rats that presented seizures, SE for over $30 \mathrm{~min}$ and that did not died within $24 \mathrm{~h}$. For neurochemical determinations of lipid peroxidation level, the rats (pilocarpine and control groups) were killed by decapitation $24 \mathrm{~h}$ after the treatment and their brains were dissected on ice to remove the striatum. The cerebral area studied was ultrasonically homogenized in $1 \mathrm{ml}$ of $0.05 \mathrm{M}$ phosphate buffer, $\mathrm{pH}$ 7.0 , and the homogenates $10 \%$ were centrifuged $(800 \mathrm{~g} / 20 \mathrm{~min}$ ) and the supernatant of the homogenates was used for lipid peroxidation level investigation. The lipid peroxidation level in the striatum during seizure activity was analyzed by measuring the thiobarbituric-acid-reacting substances (TBARS) in homogenates, as previously described by Draper and Hadley, 1990. Results are expressed as means + S.E.M. for the number of animals shown inside in parenthesis. Results were expressed as nmol of malondialdehyde (MDA)/g wet tissue. The Student-Newman-Keuls test was used for multiple comparisons of means of two groups of data. Differences in experimental groups were determined by two-tailed analysis of variance. Differences were considered significant at $p<0.05$. ${ }^{a} p<$ 0.05 as compared with control group (analysis of variance and StudentNewman-Keuls test).

(800 $\mathrm{g} / 5 \mathrm{~min})$ the TBARS were determined by the absorbance at $535 \mathrm{~nm}$. The results above were expressed as nmol of malondialdehyde (MDA)/g wet tissue.

Nitrite content determination in the striatum and frontal cortex during seizure activity. Researches verified an increase in nitrite/nitrate content in mice brain after pilocarpine-induced seizures, ${ }^{39}$ and in cerebrospinal fluid of patients with rotavirus gastroenteritis induced convulsion. ${ }^{40}$ According to this data, we decided to measure the nitrite content in rats brain during seizure activity. For the experimental procedure, homogenates $10 \%(\mathrm{w} / \mathrm{v})$ of each area investigated were prepared for both groups. The homogenates $10 \%$ were centrifuged $(800 \mathrm{~g} / 20 \mathrm{~min})$ and the supernatant of the homogenates was used for nitrite content investigation. To determine nitrite content of the control rats $(n=10)$ and pilocarpine group $(n=10)$ the homogenates 
$10 \%(\mathrm{w} / \mathrm{v})$ were centrifuged $(800 \mathrm{~g} / 10 \mathrm{~min})$. The supernatant of the homogenates was collected and the NO production was determined based on Griess reaction. ${ }^{41}$ Briefly, $100 \mu \mathrm{l}$ of supernatant was incubated with $100 \mu \mathrm{l}$ of the Griess reagent [1\% sulfanilamide in $1 \% \mathrm{H}_{3} \mathrm{PO}_{4} / 0.1 \% \mathrm{~N}$-(1-naphthyl)-ethylenediamine dihydrochloride/ $1 \% \quad \mathrm{H}_{3} \mathrm{PO}_{4} /$ distilled water, $\left.1: 1: 1: 1\right]$ at room temperature for $10 \mathrm{~min}$. The absorbance was measured at $550 \mathrm{~nm}$ via a microplate reader. Nitrite concentration was determined from a standard nitrite curve generated by using $\mathrm{NaNO}_{2}$. The results above were expressed as $\mathrm{mM}$.

Determination of glutathione reduced (GSH) concentration in the striatum and frontal cortex during seizure activity. A literature review revealed that pilocarpine-induced seizures produces a decrease in glutathione reduced (GSH) concentration in the hippocampus of rats. ${ }^{3}$ The striatum and fontal cortex are responsible by propagation and maintenance of seizure activity. Therefore, we decided to measure the GSH concentration in these areas during seizure activity. For the experimental procedure, homogenates $10 \%$ $(\mathrm{w} / \mathrm{v})$ of each area investigated were prepared for both groups. The homogenates $10 \%$ were centrifuged $(800 \mathrm{~g} / 20 \mathrm{~min})$ and the supernatant of the homogenates was used for GSH concentration investigation. GSH concentration in the pilocarpine group $(n=10)$ and control animals $(n=10)$ was analyzed. The hippocampus, striatum and frontal cortex were homogenized in 0.02 M EDTA. Immediately thereafter, $10 \%(\mathrm{w} / \mathrm{v})$ homogenates were assayed for GSH as described by Sedlak and Lindsay. ${ }^{42}$ The results above were expressed as ng/g wet tissue.

Superoxide dismutase activity determinations in the striatum and frontal cortex during seizure activity. A literature review revealed articles that address the role of antioxidant enzymes and oxidative stress in neurological disorders, including those involving different seizure models where the modulation of the pro-oxidant/ antioxidant balance by seizures per se and by antioxidant agents is discussed. However, the critical role of antioxidant enzymes in all seizure models is not uniform. Therefore, there is a need for a study about the superoxide and catalase activity during seizures induced by pilocarpine that will address this issue. ${ }^{27,33,43}$ The cerebral areas studied were ultrasonically homogenized in $1 \mathrm{ml}$ of $0.05 \mathrm{M}$ phosphate buffer, $\mathrm{pH} 7.0$, the protein concentration was measured according to the method described by Lowry et al. ${ }^{44}$ and the homogenates $10 \%$ were centrifuged $(800 \mathrm{~g} / 20 \mathrm{~min})$ and the supernatant of the homogenates was used for superoxide dismutase enzymatic activity investigation. In order to measure superoxide dismutase activity in the pilocarpine group $(n=8)$ and control animals $(n=9)$, the method employing xanthine and xanthine oxidase to generate superoxide radicals was used. ${ }^{45}$ They react with 2-4-iodophenyl-3-4-nitrophenol-5-phenyltetrazolium chloride to form a red formazan dye. The degree of the inhibition of this reaction was measured to asses' superoxide dismutase activity. The standard assay substrate mixture contained $3 \mathrm{ml}$ of xanthine $(500 \mu \mathrm{M}), 7.44 \mathrm{mg}$ of cytochrome $C, 3.0 \mathrm{ml}$ of $\mathrm{KCN}(200 \mu \mathrm{M})$, $3.0 \mathrm{ml}$ of ethylenediamine tetraacetic acid $(1 \mathrm{mM})$ in $18.0 \mathrm{ml}$ of $0.05 \mathrm{M}$ phosphate buffer, $\mathrm{pH}$ 7.0. The sample aliquot $(20 \mu \mathrm{l})$ was added to $975 \mu \mathrm{l}$ of the substrate mixture plus $5 \mu \mathrm{l}$ xanthine oxidase. After $1 \mathrm{~min}$, initial absorbance was recorded and the timer was started. Final absorbance was recorded after 6 min.
The reaction was followed at $550 \mathrm{~nm}$. Purified bovine erythrocyte superoxide dismutase Randox Laboratories, UK was used under identical conditions in order to obtain a calibration curve showing the correlation of the inhibition percentage of formazan dye formation and superoxide dismutase (SOD) activity. The SOD activity in the samples was determined from this curve. The SOD activity in the samples was determined from this curve and results expressed as $\mathrm{U} / \mathrm{mg}$ of protein.

Catalase activity determinations in the striatum and frontal cortex during seizure activity. The cerebral areas studied were ultrasonically homogenized in $1 \mathrm{ml}$ of $0.05 \mathrm{M}$ phosphate buffer, $\mathrm{pH} 7.0$, the protein concentration was measured according to the method described by Lowry et al. ${ }^{44}$ and the homogenates $10 \%$ were centrifuged $(800 \mathrm{~g} / 20 \mathrm{~min})$ and the supernatant of the homogenates was used for catalase (CAT) enzymatic activity investigation. CAT activity in the control group $(n=9)$ and pilocarpine group $(n=8)$ was measured by the method that employs hydrogen peroxide to generate $\mathrm{H}_{2} \mathrm{O}$ and $\mathrm{O}_{2}{ }^{46}$ The activity was measured by degree of this reaction. The standard assay substrate mixture contained $0.30 \mathrm{ml}$ of hydrogen peroxide in $50 \mathrm{ml}$ of $0.05 \mathrm{M}$ phosphate buffer, $\mathrm{pH}$ 7.0. The sample aliquot $(20 \mu \mathrm{l})$ was added to $980 \mu \mathrm{l}$ of substrate mixture. After $1 \mathrm{~min}$, the initial absorbance was recorded and final absorbance was read after $6 \mathrm{~min}$. The reaction was followed at $230 \mathrm{~nm}$. A standard curve was established using purified catalase (Sigma MO, USA) under identical conditions. All samples were diluted with $0.1 \mathrm{mmol} / \mathrm{l}$ phosphate buffer ( $\mathrm{pH} 7.0)$ to produce an inhibition $50 \%$ of the diluent rate (i.e., the uninhibited reaction) and results expressed as $\mathrm{mM} / \mathrm{min} / \mu \mathrm{g}$ of protein. ${ }^{47}$

Statistical analysis. Results are expressed as means \pm S.E.M. for the number of experiments, with all measurements performed in duplicate. The Student-Newman-Keuls test was used for multiple comparisons of means of two groups of data. Differences were considered significant at $\mathrm{p}<0.05$. Differences in experimental groups were determined by two-tailed analysis of variance.

\section{References}

1. Xavier SM, Barbosa CO, Barros DO, Silva RF, Oliveira AA, Freitas RM. Vitamin C antioxidant in hippocampus of adult Wistar rats after seizures and status epilepticus induced by pilocarpine. Neurosci Lett 2007; 420:76-9.

2. Barros DO, Xavier SM, Barbosa CO, Silva RF, Freitas RL, Maia FD, et al. Effects of the vitamin $\mathrm{E}$ in catalase activities in hippocampus after status epilepticus induced by pilocarpine in Wistar rats. Neurosci Lett 2007; 416:227-30.

3. Freitas RM, Vasconcelos SM, Souza FC, Viana GS, Fonteles MM. Oxidative stress in the hippocampus after status epilepticus in rats. FEBS J 2005; 272:1307-12.

4. Freitas RM, Sousa FC, Viana GS, Fonteles MM. Acetylcholinesterase activities in hippocampus, frontal cortex and striatum of Wistar rats after pilocarpine-induced status epilepticus. Neurosci Lett 2006; 399:76-8.

5. Freitas RM, Nascimento VS, Vasconcelos SM, Sousa FC, Viana GS, Fonteles MM. Catalase activity in cerebellum, hippocampus, frontal cortex and striatum after status epilepticus induced by pilocarpine in Wistar rats. Neurosci Lett 2004; 365:102-5.

6. Freitas RM, Oliveira Ade A, Vasconcelos SM, Sousa FC, Viana GS, Fonteles MM. Expression of muscarinic and dopaminergic receptors and monoamine levels frontal cortex of epileptic rats. Pharmacol Biochem Behav 2006; 83:302-6.

7. Freitas RM, Sousa FC, Vasconcelos SM, Viana GS, Fonteles MM. Pilocarpine-induced status epilepticus in rats: Lipid peroxidation level, nitrite formation, GABAergic and glutamatergic receptors alterations in the hippocampus, striatum and frontal cortex. Pharmacol Biochem Behav 2004; 78:327-30.

8. Rauca C, Wiswedel I, Zerbe R, Keilhoff G, Krug M. The role of superoxide dismutase and $\alpha$-tocopherol in the development of seizures and kindling induced by pentilenotetrazol-influence of the radical scavenger $\alpha$-phenyl-N-tert-butyl nitrone. Brain Res 2004; 1009:203-12. 


\section{Oxidative stress after pilocarpine-induced seizures}

9. Turski WA, Cavalheiro EA, Schwarz M, Czuczwar SJ, Kleinrok Z, Turski L. Limbic seizures produced by pilocarpine in rats: Behavioural, eletroencephalographic and neuropathological study. Behav Brain Res 1983; 9:315-36.

10. Cavalheiro EA, Fernandes MJ, Turski L, Naffah-Mazzacoratti MG. Spontaneous recurrent seizures in rats: Amino acid ad monoamine determination in the hippocampus. Epilepsia 1994; 35:1-11.

11. de Freitas RM, de Sousa FC, Vasconcelos SM, Viana GS, Fonteles MM. Acute alterations of neurotransmitters levels in striatum of young rat after pilocarpine-induced status epilepticus. Arq Neuropsiquiatr 2003; 61:430-3.

12. Smith BN, Shibley H. Pilocarpine-induced status epilepticus results in mossy fiber sprouting and spontaneous seizures in C57BL/6 and CD-1 mice. Epilepsy Res 2002; 49:109-20.

13. Frantseva MV, Velazquez JL, Hwang PA, Carlen PL. Free radical production correlates with cell death in a vitro model of epilepsy. Eur J Neurosci 2000; 12:1431-9.

14. Bellissimo MI, Amado D, Abdalla DS, Ferreira EC, Cavalheiro EA, Naffah-Mazzacoratti MG. Superoxide dismutase, glutathione peroxidase activities and the hydroperoxide concentration are modified in the hippocampus of epileptic rats. Epilepsy Res 2001; 46:121-8.

15. McCord JM. Superoxide radical: Controversies, contradiction and paradoxes. Proc Soc Exp Biol Med 1989; 209:112-7.

16. Ferrer I, López E, Blanco R, Rivera R, Krupinski J, Martí E. Differential c-fos and caspase expression following kainic acid excitotoxicity. Acta Neuropathol 2000; 99:24556.

17. Michiels C, Raes M, Toussaint O, Remacle J. Importance of Se-glutathione peroxidase, catalase and $\mathrm{Cu} / \mathrm{Zn}$-SOD for cell survival against oxidative stress. Free Radic Biol Med 1994; 17:235-48.

18. Erakovic V, Zupan G, Varljen J, Laginja J, Simonic A. Lithium plus pilocarpine induced status epilepticus-biochemical changes. Neurosci Res 2000; 36:157-66.

19. Vanhatalo S, Riikonen R. Cerebrospinal fluid levels of nitric oxide metabolites (nitrates/ nitrites) in infantile spasms. Epilepsia 1999; 40:210-2.

20. Vanhatalo S, Riikonen R. Nitric oxide metabolites, nitrates and nitrites in the cerebrospinal fluid in children with west syndrome. Epilepsy Res 2001; 46:3-13.

21. Rong Y, Doctrow SR, Tocco G, Baudry M. EUK-134, a synthetic superoxide dismutase and catalase mimetic, prevents oxidative stress and attenuates kainate-induced neuropathology. Proc Natl Acad Sci 1999; 96:9897-902.

22. Bruce AJ, Baudry M. Oxygen free radicals in rat limbic structures after kainate-induces seizures. Free Radic Biol Med 1995; 18:993-1002.

23. Marinho MM, de Sousa FC, de Bruin VM, Vale MR, Viana GS. Effects of lithium, alone or associated with pilocarpine, on muscarinic and dopaminergic receptors and on phosphoinositide metabolism in rat hippocampus and striatum. Neurochem Int 1998; 33:299-306

24. Costa-Lotufo LV, Fonteles MM, Lima IS, de Oliveira AA, Nascimento VS, de Bruin $\mathrm{VM}$, et al. Attenuating effects of melatonin on pilocarpine-induced seizures in rats. Comp Biochem Physiol C Pharmacol Toxicol Endocrinol 2002; 131:521-9.

25. Santos LF, Freitas RL, Xavier SM, Saldanha GB, Freitas RM. Neuroprotective actions of vitamin $\mathrm{C}$ related to decreased lipid peroxidation and increased catalase activity in adult rats after pilocarpine-induced seizures. Pharmacol Biochem Beh 2008; 89:1-5.

26. Gilbert JC, Sawas AH. ATPase activities and lipid peroxidation in rat cerebral cortex synaptosomes. Arch Int Pharmacodyn 1983; 263:189-96.

27. Hayashi M. Oxidative stress in developmental brain disorders. Neuropathology 2009; 29:1-8.

28. Günes S, Dirik E, Yis U, Seçkin E, Kuralay F, Köse S, et al. Oxidant status in children after febrile seizures. Pediatr Neurol 2009; 40:47-9.

29. Yamanaka G, Ishii C, Kawashima H, Oana S, Miyajima T, Hoshika A. Cerebrospinal fluid Diacron-Reactive Oxygen Metabolite levels in pediatric patients with central nervous system diseases. Pediatr Neurol 2008; 39:80-4.

30. Zugno AI, Stefanello FM, Scherer EB, Mattos C, Pederzolli CD, Andrade VM Guanidinoacetate decreases antioxidant defenses and total protein sulfhydryl content in striatum of rats. Neurochem Res 2008; 33:1804-10.

31. Hort J, Brozek G, Komárek V, Langmeier M, Mares P. Interstrain differences in cognitive functions in rats in relation to status epilepticus. Behav Brain Res 2000; 112:77-83.

32. Halliwell B, Gutteridge JMC. Free radicals in biology and medicine. Oxford Science Publications, London 1999; 185-7.

33. Golden TR, Patel M. Catalytic antioxidants and neurodegeneration. Antioxid Redox Signal 2008.

34. Freitas RM. Pharmacological effects of lipoic acid in behavioural alterations and in striatal superoxide dismutase activity of rats after pilocarpine-induced seizures. Rev Bras Farm 2009; in press.

35. Lapin IP, Mirzaev SM, Ryzov IV, Oxenkrug GF. Anticonvulsant activity of melatonin against seizures induced by quinolinate, kainate, glutamate, NMDA and pentylenetetrazol in mice. J Pineal Res 1998; 24:215-8.

36. Cavalheiro EA, Leite JP, Bortolotto ZA, Turski WA, Ikonomidou C, Turski L. Longterm effects of pilocarpine in rats: structural damage of the brain triggers kindling and spontaneous recurrent seizures. Epilepsia 1991; 32:778-82.
37. Walz R, Moreira JF, Benfato MS, Quevedo J, Schorer N, Vianna MR, et al. Lipid peroxidation in hippocampus early and late after status epilepticus induced by pilocarpine of kainic acid in Wistar rats. Neuroscience Letters 2000; 291:179-82.

38. Draper HH, Hadley M. Malondialdehyde determination as an index of lipid peroxidation. Methods Enzymol 1990; 186:421-31.

39. Oliveira AA, Almeida JP, Freitas RM, Nascimento VS, Aguiar LM, Júnior HV. Effects of levetiracetam in lipid peroxidation level, nitrite-nitrate formation and antioxidant enzymatic activity in mice brain after pilocarpine-induced seizures. Cell Mol Neurobiol 2007; 27:395-406

40. Kawashima H, Inage Y, Ogihara M, Kashiwagi Y, Takekuma K, Hoshika A, et al. Serum and cerebrospinal fluid nitrite/nitrate levels in patients with rotavirus gastroenteritis induced convulsion. Life Sci 2004; 74:1397-405

41. Green LC, Tannenbaum SR, Goldman P. Nitrate synthesis in the germfree and conventional rat. Science $1981 ; 212: 56-8$.

42. Sedlak J, Lindsay RH. Estimation of total, protein-bound and nonprotein sulfhydryl groups in tissue with Ellman's reagent. Anal Biochem 1968; 25:192-205.

43. Devi PU, Manocha A, Vohora D. Seizures, antiepileptics, antioxidants and oxidative stress: an insight for researchers. Expert Opin Pharmacother 2008; 9:3169-77.

44. Lowry H, Rosebrough NJ, Farr AL. Protein measurements with the folin phenol reagent. J Biol Chem 1991; 193:265-75.

45. Flohe L, Otting F. Superoxide dismutase assays. Methods Enzymol 1984; 105:93-104.

46. Chance B, Maehly AC. Assay catalases and peroxidases. Methods Enzymol 1955; 2:764-8.

47. Maehly AC, Chance B. The assay catalases and peroxidases. Methods Biochem Anal 1954; 1:357-9. 


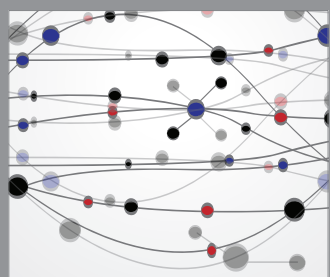

The Scientific World Journal
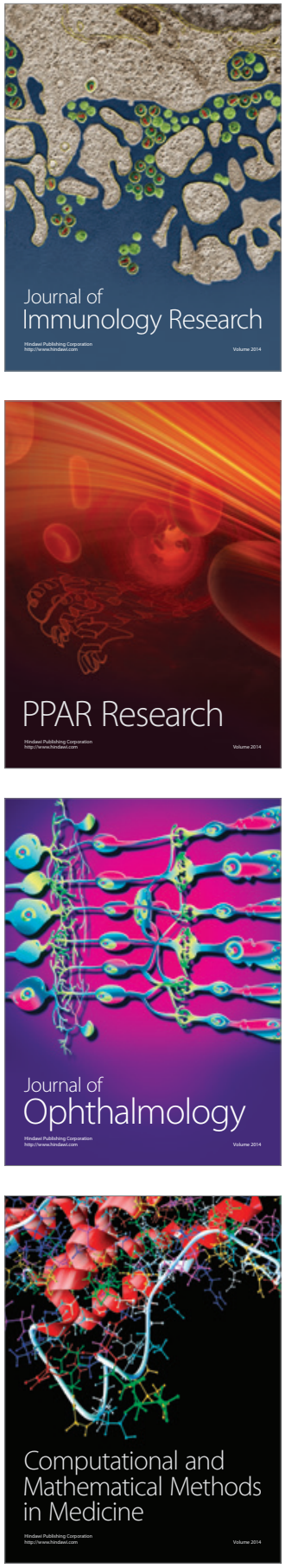

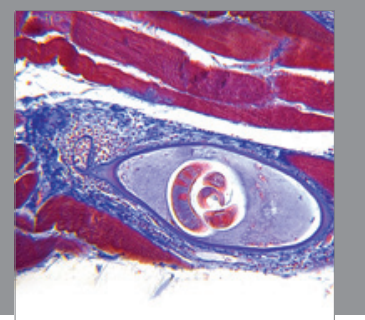

Gastroenterology

Research and Practice
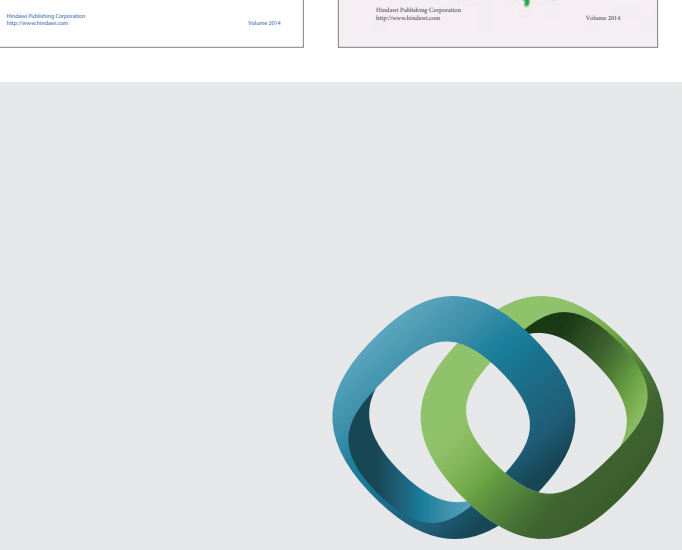

\section{Hindawi}

Submit your manuscripts at

http://www.hindawi.com
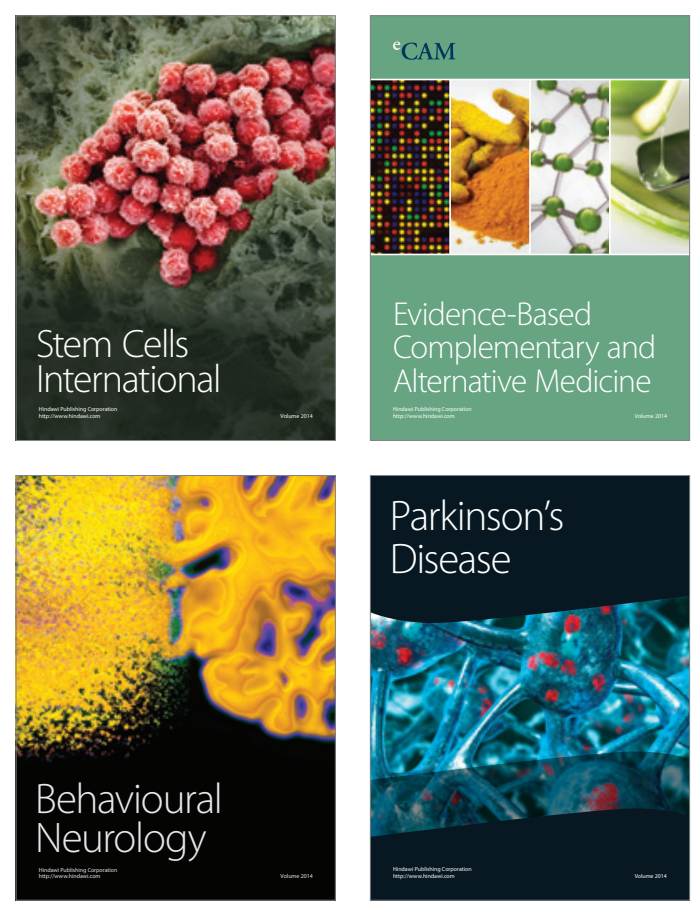

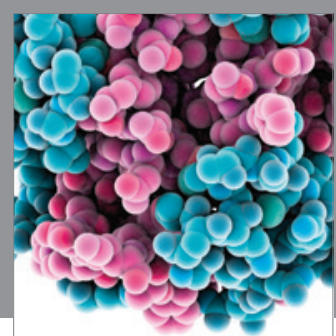

Journal of
Diabetes Research

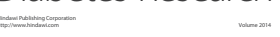

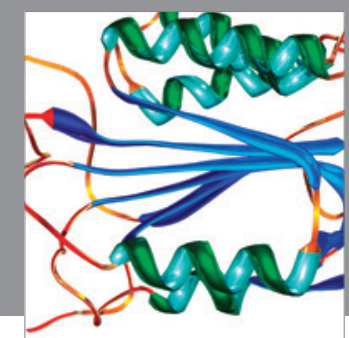

Disease Markers
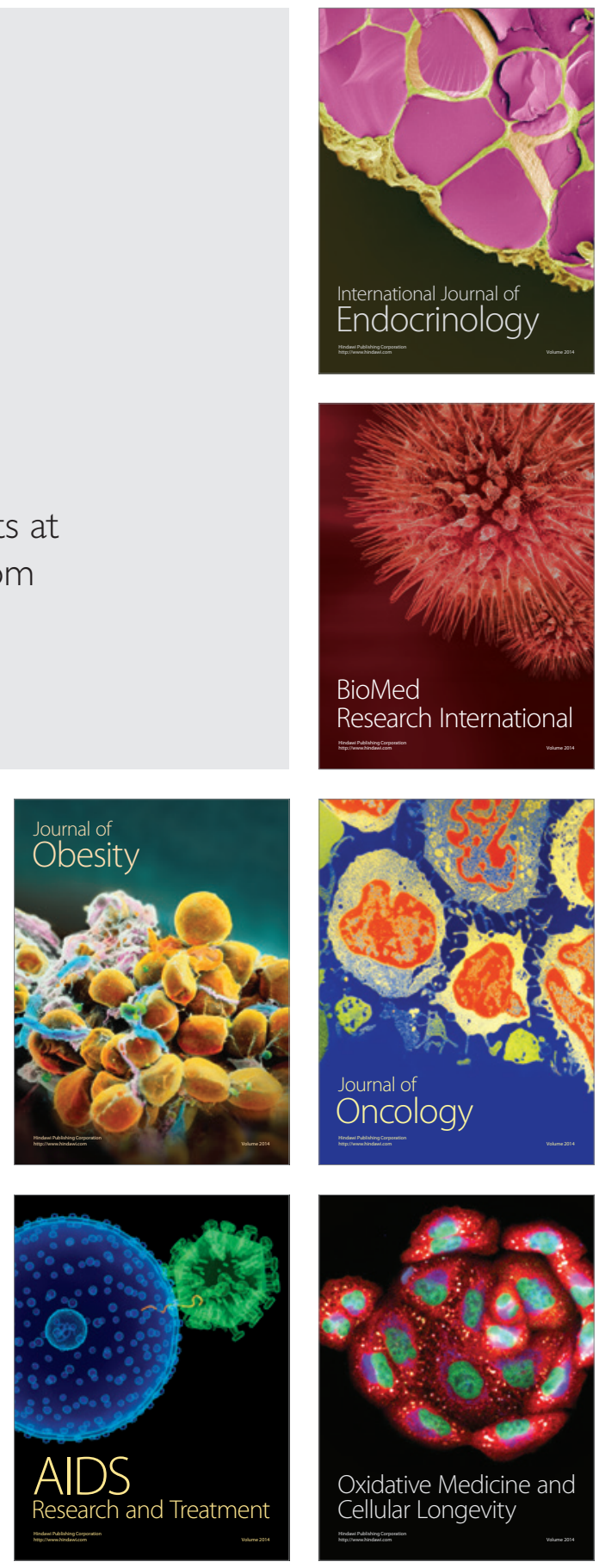